\title{
Research on Composition of Social Credibility Index Based on Artificial Intelligence Model
}

\author{
Na Liu $(D)$ and Nanke Ye \\ Public Administration, Hohai University, Nanjing 210000, China \\ Correspondence should be addressed to Na Liu; 140215050005@hhu.edu.cn
}

Received 9 July 2020; Revised 9 September 2020; Accepted 24 September 2020; Published 14 October 2020

Academic Editor: Hongju Cheng

Copyright (c) $2020 \mathrm{Na}$ Liu and Nanke Ye. This is an open access article distributed under the Creative Commons Attribution License, which permits unrestricted use, distribution, and reproduction in any medium, provided the original work is properly cited.

\begin{abstract}
Honesty refers to the fairness, justice, and responsibility of public power in social life. In order to enhance the trust of public power in society, it is necessary to analyze the social credibility indicator system. In order to achieve an accurate evaluation of social credibility, this article uses big data mining and artificial intelligence analysis methods to construct a social credibility evaluation index system for public power. First of all, this paper analyzes the distribution structure model of the public power social credibility index and analyzes the empirical mode decomposition and factor analysis of the public power social credibility evaluation data sequence in the social credibility feature distribution model. Secondly, this article constructs the public power social credit evaluation and artificial intelligence data sequence model. Using high-order cumulant features as a constraint operator, the accurate evaluation and consistent estimation of the social credibility index system of public power can be realized. The grey model is used to predict the social credibility of public power and realizes the optimized design of the evaluation model of public power social credibility. The simulation results show that the model has higher accuracy, better error convergence, and objectivity when designing the social reputation index system.
\end{abstract}

\section{Introduction}

Credibility, literally, refers to the trust power of the public. In reality, it refers to the trust that those public powers show in social life, such as fairness, justice, efficiency, humanity, democracy, and responsibility. Trust is at the heart of credibility $[1,2]$. It is an indispensable quality of social organizations, industries, departments, and government agencies and is the basis of survival and standing. Credibility as an intangible asset is gradually formed in the long-term development, reflecting an irreplaceable authority [3]. However, the credibility of the current authority, such as the justice sector, which symbolizes fairness and justice, is in some places a coalition of power. In the face of power and money, some judges can turn black and white, bend the law for selfish ends, and brazenly make false and false cases [4]. Even the most authoritative departments of industry and commerce and notaries are constantly questioned. In some places, trade and industry bureaus can openly issue certificates of competency for fake and shoddy products, making them popular in the market. Some notaries even notarized the living as dead [5]. In recent years, due to unfair notarization caused by more and more controversy, the notarization department went to the dock again and again. Such a credit-dependent industry is no longer credible, what else can be trusted? The occurrence of these events really exposed the loss of the credibility of the industry sector, causing people to pay attention to social integrity [6]. It is necessary to study a credible index evaluation system to improve the credibility of departments and promote the development of administrative departments.

Recently, the Well-off Society Research Center conducted a survey on the honesty of various social groups. The results showed that of the 49 social groups, the most honest ones included farmers, soldiers, and students. Trust in the government is plummeting [7]. Many interviewees said they no longer believed the various social survey data released by the authorities, saying the figures were false in whole or in large part. In order to promote the construction of social credibility, we need to study an effective evaluation index system of credibility [8]. Make clear the work responsibility and 
function orientation of the public power's social credibility, ensure the coordination of the public power's social credibility work, so that the role of the organization's various departments can be brought into full play, the evaluation of public power's social credibility is based on the analysis of big data and artificial intelligence, and it can take the public power's social credibility as the platform of information transmission and interaction [9].

The index system of social credibility is constructed by using big data analysis method, and the evaluation of social credibility is realized with the method of data prediction and evaluation. This paper puts forward a social credibility index structure and evaluation system based on the artificial intelligence model, and it uses big data mining and artificial intelligence analysis method to construct the social credibility evaluation index system of the public power [10]. This paper analyzes the distribution structure model of the social credibility index of public power and analyzes the empirical modal decomposition and factor analysis of the social credibility evaluation data series of public power in the distribution model of social credibility characteristic distribution. The data sequence model of public power's social credibility evaluation and analysis of artificial intelligence is constructed [11]. Big data mining and artificial intelligence analysis are used to extract the features of the performance quantization sequence, and the higher-order cumulant feature is used as the postconvergence operator. To realize the accurate evaluation and consistency estimation of the social credibility index system of public power [12]. The Grey model is used to predict the social credibility of public power, and the optimization design of public power social credibility evaluation model is realized. Finally, the performance test is carried out through the simulation experiment, which shows the superior performance of this method in improving the accuracy of social credibility evaluation.

\section{Data Analysis and Preprocessing Social Credibility Evaluation Model}

2.1. Analysis of the Quantitative Evaluation Data of Public Power's Social Credibility. In order to realize the accurate evaluation of the public power's social credibility, the quantitative evaluation of the public power's social credibility is carried out by using the statistical characteristic sequence analysis method [12]. Construct the data transmission structure model of the public power's social credibility quantitative evaluation statistical characteristic sequence; Quantitative evaluation of social credibility of public power the mathematical evolution model of statistical feature sequence can be constructed according to the quantitative characteristics such as historical evaluation data of public power social credibility [13].

Based on the above analysis, the artificial intelligence analysis flow of the social credibility index evaluation system designed in this paper is shown in Figure 1.

The management analysis is carried out on the evolvement object structure of public power's social credibility. The ontology model of social credibility evaluation is obtained, and the transmission structure model of the statistical characteristic sequence of quantitative evaluation of

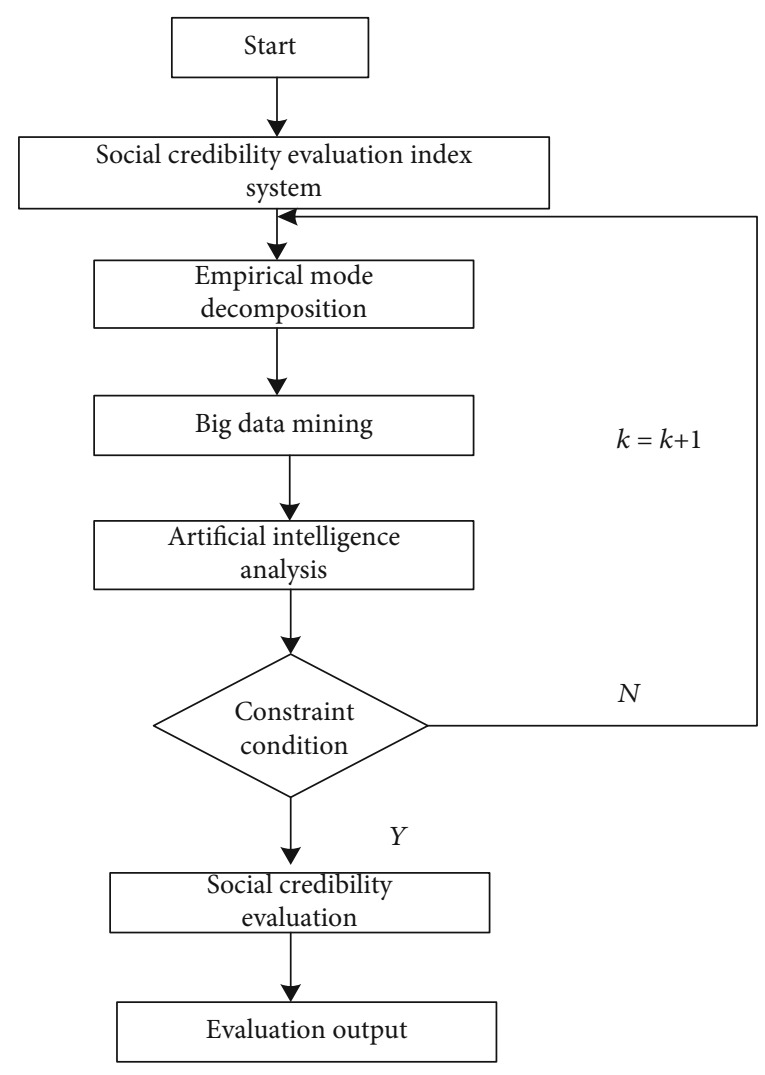

FIGURE 1: Artificial intelligence analysis process of social credibility index evaluation system.

social credibility of public power expressed by the directed label graph is $O=\left(C, I, P, H c, R, A^{0}\right)$. Among them, two groups of ontology fragment set $G(O)=\left(V, E, L_{V}, L_{E}, \mu, \eta\right)$ and edge set $V=C$ of social credibility evaluation, $E=V \times$ $V$ is the mapping function of two ontology models of social credibility evaluation as input. According to the mapping of the correlation sets of two index systems of social credibility evaluation, the feature extraction is carried out with an adaptive learning method [14]. At present, the fuzzy degree and compactness index of public power's social credibility evaluation is $\left(R T_{1}, R T_{2}\right)$, and the quantitative set of public power evaluation index satisfies the constraint function $[15,16]$ :

$$
F_{j}=\sum_{k=1}^{n} X_{k j}, Q_{j}=\sum_{k=1}^{n}\left(X_{k j}\right)^{2}
$$

According to the above description, the quantitative evaluation of the social credibility of public power is obtained [17]. The result of the directed marker graph structure analysis of the statistical feature sequence data is obtained, and the social credibility index of the public power is calculated as the semantic ontology characteristic state of the information flow of the public power system $[18,19]$ :

$$
x_{n}=x\left(t_{0}+n \Delta t\right)=h\left[z\left(t_{0}+n \Delta t\right)\right]+\omega_{n} .
$$

In which, $M$ is a directed marker graph structure window 
feature function of $d$ dimension, and the geometric invariant of the statistical feature sequence of public power's social credibility is calculated [20]. The interference vector model of the data on the object structure of the social credibility evaluation of public power is expressed as follows $[5,21]$ :

$z(t)=s(t)+j s(t) \otimes h(t)=s(t)+j \int_{-\infty}^{+\infty} \frac{s(u)}{t-u} d u=s(t)+j H[s(t)]$.

The relevant function of quantitative evaluation of social credibility of public power in statistical feature series data mining is constructed as:

$$
X=\left\{x_{1}, x_{2}, \cdots x_{n}\right\} \subset R^{s}
$$

The index system of public power's social credibility can be regarded as a series of nonlinear statistical characteristics, and the trend of public power's social credibility is analyzed by the method of nonlinear statistical characteristic sequence analysis [7]. Based on the statistical analysis of the social credibility of public power, the fitting state model of describing the social credibility of public power by using a multivariate statistical characteristic equation is obtained as follows:

$$
\left(\begin{array}{c}
X \\
P(X)
\end{array}\right)=\left\{\begin{array}{cccc}
a_{1}, & a_{2}, & \cdots, & a_{m} \\
p\left(a_{1}\right), & p\left(a_{2}\right), & \cdots, & p\left(a_{m}\right)
\end{array}\right\} .
$$

In which, $0 \leq p\left(a_{i}\right) \leq 1(i=0,1,2, \cdots, m)$ and $\sum_{i=1}^{m} p\left(a_{i}\right)=1$, the autoregressive statistical characteristic parameters representing the social credibility of public power, $a_{i i}$ are obtained by decomposing the covariance matrix of the solution vector of the statistical equation, and the principal component of the statistical characteristic information is obtained [8]. In the feature space of the social credibility distribution, the entropy of the distribution characteristic information of the public power's social credibility is obtained through the discrete and analytic processing of the data [9]:

$$
H(X)=E\left(I\left(a_{i}\right)\right)=-\sum_{i=1}^{m} p\left(a_{i}\right) \log _{2} p\left(a_{i}\right)
$$

On the basis of the above analysis, the collection of quantitative evaluation data of social credibility of public power and the reorganization of distributed structure are realized, which provides an accurate data input basis for social credibility evaluation and prediction [10].

2.2. Feature Decomposition and Preprocessing of Evaluation Data of Society. In the social credibility characteristic distribution model, the social credibility evaluation data sequence of public power is decomposed by empirical mode decomposition and characteristic decomposition, and the social credibility evaluation data sequence model and artificial intelligence analysis are carried out, and the public power's social credibility system is distributed as a two-order system [11]. Using the stochastic analysis model, we get the public credibility test data sequence $x$ of the public power. The expression of the auxiliary spatial test cumulative $j$ is

$$
\text { Corr }=\frac{\left\langle\left(x_{n}-\bar{x}\right)\left(x_{n-d}-\bar{x}\right)\left(x_{n-D}-\bar{x}\right)\right\rangle}{\left\langle\left(x_{n}-\bar{x}\right)^{3}\right\rangle} .
$$

In which, $x_{n}$ represents the statistical element of public power's social credibility information $D=2 d, d$ indicates the sampling statistical delay of public power's social credibility, SD indicates the predictor of public power's social credibility, and $\langle x(n)\rangle$ represents the mean value of $x(n)$ :

$$
\langle x(n)\rangle=1 / N \sum_{n=1}^{N} x(n)
$$

For a continuous public power social credibility evaluation data series, each spatial solution vector of the social credibility of public power training subset $S_{i}(i=1,2, \cdots, L)$, statistical feature analysis, and regional differential equation description common are used, and the empirical mode decomposition process of social credibility of common power is expressed as follows [12]:

$$
\begin{gathered}
c_{1 x}(\tau)=E\{x(n)\}=0, \\
c_{2 x}(\tau)=E\{x(n) x(n+\tau)\}=r(\tau), \\
c_{k x}\left(\tau_{1}, \tau_{2}, \cdots, \tau_{k-1}\right) \equiv 0(k \geq 3) .
\end{gathered}
$$

While $q=2$, the social credibility information vector of the public power satisfies the convergence condition of the constraint function of the differential equation, and the constraint characteristic decomposition formula of the social credibility is obtained as follows:

$$
\Psi_{x}(\omega)=\ln \Phi_{x}(\omega)=-\frac{1}{2} \omega^{2} \sigma^{2}
$$

Based on the factor analysis of the social credibility of public power, the characteristics of association rules are obtained as follows:

$$
g\left(x_{i}, y_{j} \mid \mu_{k}, \sigma_{k}^{2}\right)=\prod_{k=1}^{K} a_{k} \frac{1}{\sqrt{2 \pi \sigma_{k}^{2}}} \exp \left\{-\frac{\left(x_{i}-\mu_{k}\right)^{2}}{2 \sigma_{k}^{2}}\right\} .
$$

In which, $a_{i j} \in \boldsymbol{G} \boldsymbol{F}\left(2^{n}\right)$ is the vector field of the data sequence of social credibility evaluation of public power under the condition of global gradual stability, and $u \mapsto u_{\lambda}$ is the descriptive statistical characteristic value of social credibility [12].

\section{Index Composition and Evaluation Model Optimization of Social Credibility}

Big data mining and artificial intelligence analysis are used to extract the features of social credibility quantitative series [22]. A time-dimensional quantitative evaluation of the 
social credibility of public power is introduced in this paper. Using $F^{2 \alpha / \pi}=\sum_{i=0}^{3} a_{i}(\alpha) W^{i}$ to calculate the social credibility of public power, the characteristic value and eigenvector of statistical feature series are evaluated, and $S=\sqrt{\Delta t \Delta f}$ is chosen to decompose into a fractional Fourier transform scale, and the common value is obtained by artificial intelligence analysis method [23]. Quantitative Evaluation of the Social credibility of the Common Power the sequence of statistical features $x(t)[24]$ :

$$
x(t)=g(t) \exp \left[-j \pi t^{2} \tan (\alpha / 2)\right] .
$$

The interval length of the statistical characteristic sequence $x(t)$ is equal to the dimensionless quantity, that is $\Delta x=\sqrt{\Delta t \Delta f}$, the two intervals are normalized to $[-\Delta x / 2, \Delta$ $x / 2]$. The artificial intelligence analysis method is adopted to calculate the statistical characteristic sequence of the social credibility index under a single random variable, which comprises the following steps [25]:

$x(t) \mathrm{e}^{j \pi t^{2} \cot \alpha}=\sum_{n=-N}^{N} x\left(\frac{n}{2 \Delta x}\right) \mathrm{e}^{\left(j \pi(\cot \alpha) n^{2} /(2 \Delta x)^{2}\right)} \sin c\left[2 \Delta x\left(t-\frac{n}{2 \Delta x}\right)\right]$.

A set of orthogonal chirp bases is used to expand the space of characteristic function of social credibility index, and the data envelopment feature of social credibility index is obtained [26]:

$$
\begin{aligned}
X_{p}(u)= & A_{\alpha} \exp \left(j \pi(\cot \alpha) u^{2}\right) \sum_{n=-N}^{N} x\left(\frac{n}{2 \Delta x}\right) \exp \left[\frac{j \pi(\cot \alpha) n^{2}}{(2 \Delta x)^{2}}\right] \\
& \times \int_{-\infty}^{\infty} \exp [-j 2 \pi(\csc \alpha) u t] \sin c\left[2 \Delta x\left(t-\frac{n}{2 \Delta x}\right)\right] d t
\end{aligned}
$$

The integral term in the upper expression is equal to [27]:

$$
\exp \left[\frac{-j 2 \pi(\csc \alpha) x n}{2 \Delta x}\right] \frac{1}{2 \Delta x} \operatorname{rect}\left[\frac{(\csc \alpha) x}{2 \Delta x}\right] \text {. }
$$

Quantitative evaluation of the social credibility of public power the second derivative of the second characteristic function $\operatorname{rect}[(\csc \alpha) x / 2 \Delta x]$ in the support region $|x| \leq \Delta x / 2$ of the transformation function can be expressed as [28]:

$X_{p}\left(\frac{m}{2 \Delta x}\right)=\frac{A_{\alpha}}{2 \Delta x} \sum_{n=-N}^{N} \mathrm{e}^{j \pi(\cot \alpha) m^{2}-j 2 \pi(\csc \alpha) m n+j \pi(\cot \alpha) n^{2} /(2 \Delta x)^{2}} x\left(\frac{n}{2 \Delta x}\right)$.

In the reconstructed quantitative sequence of social credibility of public power, any point in the evolution of social credibility is expressed as $\boldsymbol{X}_{\eta(n)}$, and the nearest neighbor in the high-dimensional Grey model of social credibility of public power is expressed as $\boldsymbol{X}_{n}$, using a large number [22]. According to mining and artificial intelligence analysis

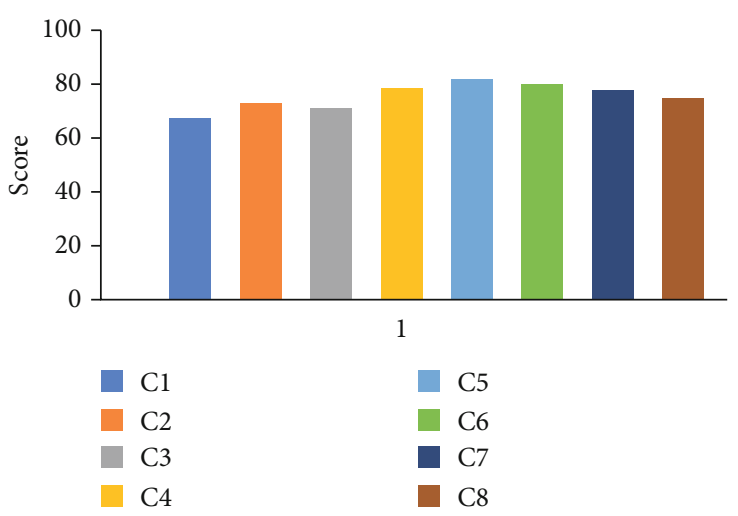

FIgURE 2: Statistical big data analysis of social credibility evaluation.

methods, the feature extraction of the performance quantization sequence is carried out, and the higher-order cumulant feature is used as the postconvergence operator to realize the accurate evaluation and consistency estimation of the social credibility index system of public power. Finally, the model is constructed and optimized according to the social credibility index.

\section{Simulation Experiment and Result Analysis}

In order to test the practical application performance of this algorithm in the analysis and evaluation of the public power's social credibility index, the simulation experiment is carried out. The experiment uses the MATLAB simulation tool to analyze the data and public rights.

The test sample set of social credibility of force comes from the national statistical department. The time of data collection is from 30 April 2017 to 5 June 2018, the weight of artificial intelligence analysis is 0.23 , the correlation coefficient is 1.24 , and the social credibility index is evaluated. The number of iterations is 10000 . According to the above simulation parameters, the social credibility is evaluated. In the simulation experiment, the data sampling result of Figure 2 is used as the research object to realize the research and evaluation of the credibility index. The confidence level comparison result obtained by the credibility evaluation is shown in Figures 3 and 4 .

Through the above experimental results, it can be observed that with the increase of the number of iterative steps, the error of social credibility evaluation is reduced, and the prediction error of public power in this paper is smaller than that of the traditional method, and the social credibility of public power is lower than that of traditional method. The clustering and fusion of data and information improves the accuracy of prediction, promotes the reform and upgrading of social functional departments, and enhances the public credibility.

\section{Conclusions}

In order to realize the accurate evaluation of social credibility, a social credibility index structure and evaluation system based on the artificial intelligence model is proposed. Big 


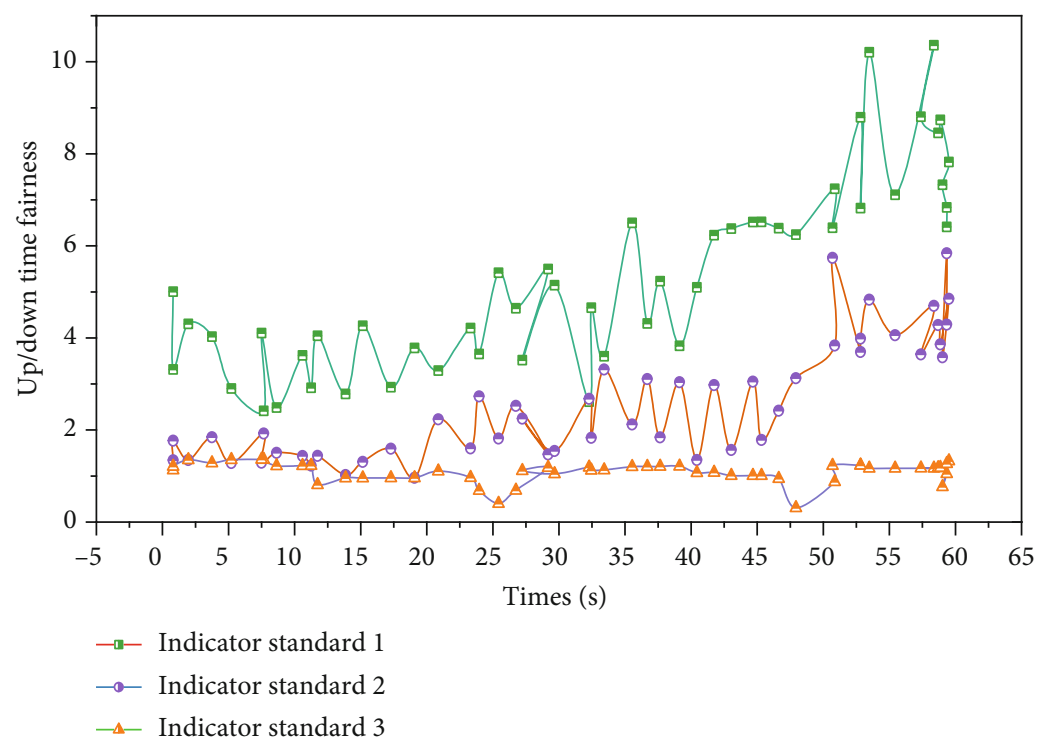

FIgURE 3: Confidence and time comparison of credibility assessment.

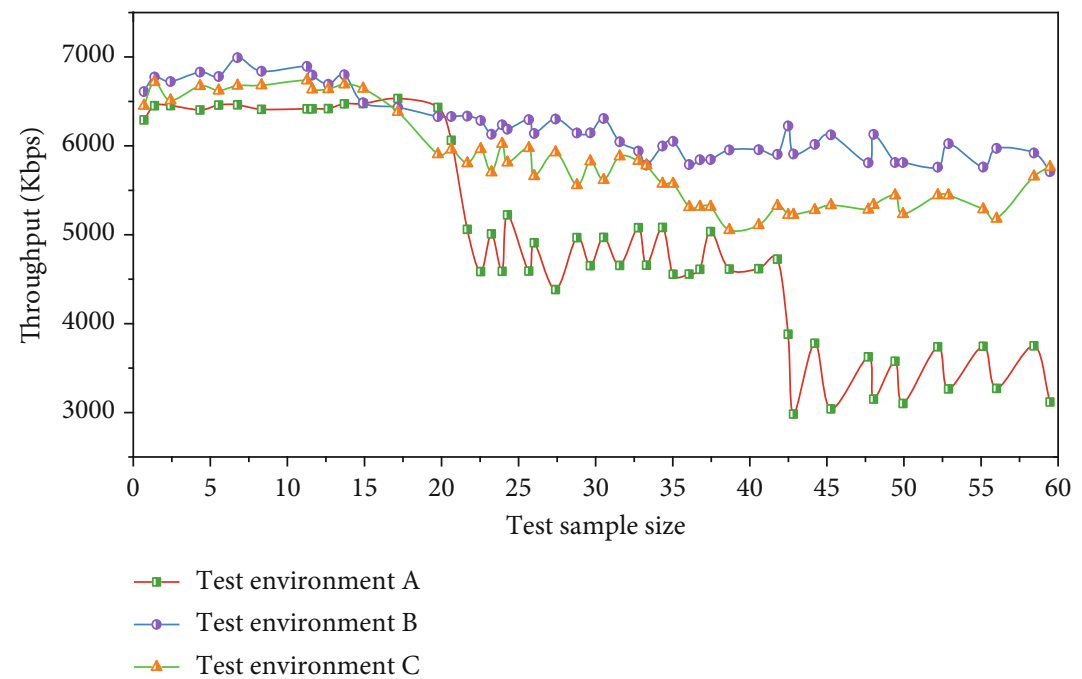

Figure 4: Network test effect diagram of social credibility index system.

data mining and artificial intelligence analysis method are used to construct the social credibility evaluation index system of the public power. This paper analyzes the distribution structure model of the social credibility index of public power and analyzes the empirical modal decomposition and factor analysis of the social credibility evaluation data series of public power in the distribution model of social credibility characteristic distribution. Construct the data sequence model of public power's social credibility evaluation and analysis of artificial intelligence.

Big data mining and artificial intelligence analysis are used to extract the feature of the performance quantization sequence. The high order cumulant feature is used as the constraint operator to realize the accurate evaluation and consistency estimation of the public power's social credibility index system. The Grey model is used to predict the social credibility of public power, and the optimization design of public power social credibility evaluation model is realized. The simulation results show that the model has higher accuracy, better error convergence, and objectivity in the design of the social credibility index system. This method has good application value in social credibility evaluation and index analysis.

\section{Data Availability}

All experimental data can be contacted by the corresponding author.

\section{Conflicts of Interest}

The authors declare that there is no conflict of interest regarding the publication of this paper. 


\section{References}

[1] W. Meng, D. Yang, and H. Huang, "Prediction of China's sulfur dioxide emissions by discrete grey model with fractional order generation operators," Complexity, vol. 2018, Article ID 8610679, 13 pages, 2018.

[2] G. F. Fan, A. Wang, and W. C. Hong, "Combining grey model and self-adapting intelligent grey model with genetic algorithm and annual share changes in natural gas demand forecasting," Energies, vol. 11, no. 7, 2018.

[3] B. Zeng and C. Li, "Forecasting the natural gas demand in China using a self-adapting intelligent grey model," Energy, vol. 112, pp. 810-825, 2016.

[4] Y. Yuan, H. Zhao, X. Yuan, L. Chen, and X. Lei, “Application of fractional order-based grey power model in water consumption prediction," Environmental Earth Sciences, vol. 78, no. 8, 2019.

[5] C. L. Spash and I. Aslaksen, "Re-establishing an ecological discourse in the policy debate over how to value ecosystems and biodiversity," Journal of Environmental Management, vol. 159, pp. 245-253, 2015.

[6] C. Shao, G. L. Ciampaglia, O. Varol, K. C. Yang, A. Flammini, and F. Menczer, "The spread of low-credibility content by social bots," Nature Communications, vol. 9, no. 1, 2018.

[7] B. A. Nosek and D. Lakens, "Registered reports: a method to increase the credibility of published results," Social Psychology, vol. 45, no. 3, pp. 137-141, 2014.

[8] X. Lin, P. R. Spence, and K. A. Lachlan, "Social media and credibility indicators: the effect of influence cues," Computers in Human Behavior, vol. 63, pp. 264-271, 2016.

[9] J. Kirchherr, H. Pohlner, and K. J. Charles, "Cleaning up the big muddy: a meta-synthesis of the research on the social impact of dams," Environmental Impact Assessment Review, vol. 60, pp. 115-125, 2016.

[10] J. Imlawi, D. Gregg, and J. Karimi, "Student engagement in course-based social networks: the impact of instructor credibility and use of communication," Computers \& Education, vol. 88, pp. 84-96, 2015.

[11] T. Hoang, J. Liu, N. Pratt et al., "Authenticity and credibility aware detection of adverse drug events from social media," International Journal of Medical Informatics, vol. 120, pp. 101-115, 2018.

[12] N. Hajli, "Ethical environment in the online communities by information credibility: a social media perspective," Journal of Business Ethics, vol. 149, no. 4, pp. 799-810, 2018.

[13] M. de los Ángeles Fernandez, M. de los Ángeles Sanromán, S. Marks et al., "A grey box model of glucose fermentation and syntrophic oxidation in microbial fuel cells," Bioresource Technology, vol. 200, pp. 396-404, 2016.

[14] J. Best, "The inflation game: targets, practices and the social production of monetary credibility," New Political Economy, vol. 24, no. 5, pp. 623-640, 2019.

[15] N. C. Benda, L. T. Das, E. L. Abramson et al., “"How did you get to this number?" Stakeholder needs for implementing predictive analytics: a pre-implementation qualitative study," Journal of the American Medical Informatics Association, vol. 27, no. 5, pp. 709-716, 2020.

[16] S. Aladhadh, X. Zhang, and M. Sanderson, "Location impact on source and linguistic features for information credibility of social media," Online Information Review, vol. 43, no. 1, pp. 89-112, 2019.
[17] S. Bi, "Intelligent system for english translation using automated knowledge base," Journal of Intelligent and Fuzzy Systems, vol. 5, pp. 1-10, 2020.

[18] Y. Gui and G. Zeng, "Joint learning of visual and spatial features for edit propagation from a single image," The Visual Computer, vol. 36, no. 3, pp. 469-482, 2020.

[19] Y. Wang, Z. Shen, and Y. Jiang, "Analyzing maternal mortality rate in rural China by Grey-Markov model," Medicine, vol. 98, no. 6, article e14384, 2019.

[20] X. Xiao, M. J. Hui, Z. Liu, and W. R. Qiu, "iCataly-PseAAC: identification of enzymes catalytic sites using sequence evolution information with grey model GM $(2,1)$," Journal of Membrane Biology, vol. 248, no. 6, pp. 1033-1041, 2015.

[21] B. Urrutikoetxea Arrieta, A. I. Polo Pena, and C. Martinez Medina, "The moderating effect of blogger social influence and the reader's experience on loyalty toward the blogger," Online Information Review, vol. 43, no. 3, pp. 326-349, 2019.

[22] D. Jiang, G. Li, Y. Sun, J. Kong, and B. Tao, "Gesture recognition based on skeletonization algorithm and CNN with ASL database," Multimedia Tools and Applications, vol. 78, no. 21, pp. 29953-29970, 2019.

[23] Y. He, G. Li, Y. Liao et al., "Gesture recognition based on an improved local sparse representation classification algorithm," Cluster Computing, vol. 22, no. S5, pp. 10935-10946, 2019.

[24] W. Cheng, Y. Sun, G. Li, G. Jiang, and H. Liu, "Jointly network: a network based on CNN and RBM for gesture recognition," Neural Computing and Applications, vol. 31, no. S1, pp. 309323, 2019.

[25] Y. Sang, H. Shen, Y. Tan, and N. Xiong, "Efficient protocols for privacy preserving matching against distributed datasets," International Conference on Information and Communications Security, vol. 4307, 2006.

[26] F. Long, N. Xiong, A. V. Vasilakos, L. T. Yang, and F. Sun, “A sustainable heuristic QoS routing algorithm for pervasive multi-layered satellite wireless networks," Wireless Networks, vol. 16, no. 6, pp. 1657-1673, 2010.

[27] J. Li, N. Xiong, J. H. Park, C. Liu, S. MA, and S. E. Cho, "Intelligent model design of cluster supply chain with horizontal cooperation," Journal of Intelligent Manufacturing, vol. 23, no. 4, pp. 917-931, 2012.

[28] Z. Liu, B. Hu, B. Huang, L. Lang, H. Guo, and Y. Zhao, "Decision optimization of low-carbon dual-channel supply chain of auto parts based on smart city architecture," Complexity, vol. 2020, no. 5, Article ID 2145951, p. 14, 2020.

[29] L. Dong, Q. Guo, and W. Wu, "Speech corpora subset selection based on time-continuous utterances features," Journal of Combinatorial Optimization, vol. 37, no. 4, pp. 1237-1248, 2019. 